\title{
Predicting survival in out-of-hospital cardiac arrest patients undergoing targeted temperature management: The Polish Hypothermia Registry Risk Score
}

Łukasz Kołtowski ${ }^{1}$, Beata Średniawa ${ }^{2}$, Agnieszka Tycińska ${ }^{3}$, Magdalena Czajkowska ${ }^{4}$ Magdalena Niedziela ${ }^{1,5}$, Wiesław Puchalski ${ }^{6}$, Ewa Szczerba ${ }^{1}$, Robert Kowalik ${ }^{1}$, Robert Ryczek ${ }^{7}$, Barbara Zawiślak ${ }^{8}$, Elżbieta Kremis ${ }^{9}$, Konrad Koza ${ }^{10}$, Agnieszka Nazaruk ${ }^{11}$, Joanna Wolska ${ }^{4}$, Michał Ordak ${ }^{12}$, Grzegorz Opolski ${ }^{1}$, Janina Stępińska ${ }^{9}$

${ }^{1} 1^{\text {st }}$ Department of Cardiology, Medical University of Warsaw, Poland; ${ }^{2}$ Department of Cardiology, Medical University of Silesia SMDZ, Silesian Center for Heart Diseases, Zabrze, Poland; ${ }^{3}$ Department of Cardiology, Medical University of Bialystok, Poland $;{ }^{4}$ Clinical Department of Anaesthesiology and Intensive Care, Regional Specialist Hospital, University of Warmia and Mazury, Olsztyn, Poland; ${ }^{5}$ Department of Experimental and Clinical Physiology, Center for Preclinical Research and Technology (CePT), Medical University of Warsaw, Poland; ${ }^{6} 1^{\text {st }}$ Department of Cardiology, Medical University of Gdansk, Poland; ${ }^{7}$ Department of Cardiology and Internal Diseases, Military Medical Institute, Warsaw, Poland; ${ }^{8} 2^{\text {nd }}$ Department of Cardiology and Cardiovascular Interventions, University Hospital of Krakow, Poland; ${ }^{9}$ Intensive Cardiac Therapy Clinic, Institute of Cardiology Warsaw, Poland;

${ }^{10}$ Department of Anaesthesiology and Intensive Care, Specialist Hospital, Siedlce, Poland;

${ }^{11}$ Department of Invasive Cardiology, Central Clinical Hospital of Ministry of Interior and Administration, Warsaw, Poland; ${ }^{12}$ Department of Pharmacodynamics, Center for Preclinical Research and Technology (CePT), Medical University of Warsaw, Poland

\begin{abstract}
Background: Prompt reperfusion and post-resuscitation care, including targeted temperature management (TTM), improve survival in out-of-hospital cardiac arrest (OHCA) patients. To predict inhospital mortality in OHCA patients treated with TTM, the Polish Hypothermia Registry Risk Score (PHR-RS) was developed. The use of dedicated risk stratification tools may support treatment decisions. Methods: Three hundred seventy-six OHCA patients who underwent TTM between 2012 and 2016 were retrospectively analysed and whose data were collected in the Polish Hypothermia Registry. A multivariate logistic regression model identified a set of predictors of in-hospital mortality that were used to develop a dedicated risk prediction model, which was tested for accuracy.

Results: The mean age of the studied population was $59.2 \pm 12.9$ years. $80 \%$ of patients were male, 73.8\% had shockable rhythms, and mean time from cardiac arrest (CA) to cardiopulmonary resuscitation (CPR) was $7.2 \pm 8.6 \mathrm{~min}$. The inputs for PHR-RS were patient age and score according to the Mild Therapeutic Hypothermia (MTH) Scale. Criteria for the MTH score consisted of time from CA to CPR above $10 \mathrm{~min}$, time from $C A$ to the return of spontaneous circulation above $20 \mathrm{~min}$, in-hospital CA, unwitnessed CA, and non-shockable rhythm, each counted as 1 point. The predictive value of PHR-RS was expressed as an area under the curve of 0.74.

Conclusions: PHR-RS is one of the simplest and easiest models to use and enables a reliable prediction of in-hospital mortality in OHCA patients treated with TTM. (Cardiol J 2021; 28, 1: 95-100)

Key words: targeted temperature management, temperature control, post-resuscitation care, risk prediction model
\end{abstract}

Address for correspondence: Łukasz Kołtowski, MD, PhD, $1^{\text {st }}$ Department of Cardiology, Medical University of Warsaw, ul. Banacha 1a, 02-097 Warszawa, Poland, tel: +48 2259919 51, fax: +48 22599 19 51, e-mail: lukasz@koltowski.com 


\section{Introduction}

It is estimated that each year one in 3000 individuals will suffer from out-of-hospital cardiac arrest (OHCA). Despite the implementation of an emergency system with rapid ambulance dispatch, standardization of resuscitation, advanced life support, access to prompt invasive reperfusion techniques and post-resuscitation care including targeted temperature management (TTM), the discharge survival rate among OHCA patients is barely $24 \%$ [1]. Besides TTM, other timeand resource-consuming techniques, which have shown survival benefit in selected patients, can be implemented during an intensive care unit (ICU) stay. This increasing number of treatment options with preventive potential, together with limited resources, force physicians to make choices based on subjective judgments and available general ICU risk estimation tools. Although there are several scoring tools to evaluate in-hospital mortality risk of post-cardiac arrest (CA) patients admitted to ICU, were developed before TTM became widely used. Thus, their predictive value in actively cooled OHCA patients is limited [2,3]. In order to support physicians and optimize treatment decisions in this cohort of patients, a dedicated risk score is needed. Therefore, the aim of this research project was to develop a risk prediction model for the in-hospital mortality of post-CA patients treated with TTM.

\section{Methods}

\section{Study design and study population}

The clinical data of 376 patients who underwent TTM between 2012 and 2016 and were collected in the Polish Hypothermia Registry were retrospectively analysed. The Polish Hypothermia Registry is a project initiated by the Polish Society of Cardiology and coordinated by the Medical University of Warsaw, Poland. The study was approved by the local Bioethics Committee. The nature of the registry required all centers to enter clinical data of patients prospectively and anonymously, using a web-based secured panel connected to a central database. There were no specific exclusion criteria, and all patients who received TTM $(\mathrm{n}=376)$ were included in the analysis. The collected data included demographic information and peri-event information, including time to cardiopulmonary resuscitation (CPR), time to return of spontaneous circulation (ROSC), presence of witnesses, comorbidities, Glasgow Coma Scale (GCS) score, index diagnosis, need for percutaneous coronary intervention (PCI), use of early cooling, type of stationary cooling technique, incidence of shock at each time-point throughout the in-hospital course, duration of cooling, complications, early outcome, duration of hospital stay, and clinical and neurological outcome at discharge.

\section{Definitions and standard care procedures}

All participating centres used a dedicated TTM protocol (Suppl. Appendix 1). Any patient admitted due to OHCA who met the inclusion criteria (ROSC, GCS below 8 points, time from CPR below $4 \mathrm{~h}$ ) was qualified for TTM. The survivors were assessed using the Mild Therapeutic Hypothermia (MTH) Scale which was developed to identify patients at increased risk of TTM-associated adverse events. It consists of 5 elements, each counted as 1 point (Table 1 ). To exclude intracerebral bleeding if suspected, a brain computed tomography (CT) scan was performed. All patients in whom a cardiac cause of CA was suspected underwent a coronary angiogram followed by PCI if indicated. The first cooling phase was initiated simultaneously with PCI using intravenous cool fluids and continued with stationary cooling systems (surface or endovascular) in the ICU. The targeted temperature of $32-36^{\circ} \mathrm{C}$ was maintained for $24-36 \mathrm{~h}$. After rewarming and extubating, patients who remained hemodynamically stable were transferred to the general ward.

\section{Statistical analysis and identification of predictors}

The statistical analysis was conducted with IBM SPSS Statistics 24. Continuous variables with a normal distribution are expressed as the mean \pm standard deviation (SD) and those with a skewed distribution as the median with interquartile range (IQR). Categorical variables are presented as absolute values and percentages. The Wilcoxon signedrank test was used to compare pairs of related samples, and the Pearson correlation coefficient was used for continuous variables. The adequacy of the analysed variables was assessed by fitting corresponding models and investigating the area under the receiver operating characteristic (ROC) curve (AUC). All tests were 2 -sided, and the level of significance was set at 0.05 .

\section{Results}

\section{Group characteristics}

Three hundred seventy-six post-CA patients treated with TTM were included in the study. Most 
Table 1. Mild Therapeutic Hypothermia score.

\begin{tabular}{lc}
\hline Variable & Value \\
\hline Cardiac arrest to CPR above 10 min & +1 point \\
Time from cardiac arrest to ROSC & +1 point \\
above 20 min & +1 point \\
In-hospital cardiac arrest & +1 point \\
Unwitnessed cardiac arrest & +1 point \\
Non-shockable rhythm & \\
\hline
\end{tabular}

$\mathrm{CPR}$ - cardiopulmonary resuscitation; ROSC - return of spontaneous circulation

of them were men $(\mathrm{n}=301 ; 80.1 \%$; $\mathrm{p}<0.001)$. The mean age of the studied population was $59.2 \pm 12.9$ years. One out of 5 patients had a history of myocardial infarction $(\mathrm{n}=78 ; 20.7 \%)$, and almost half of the group suffered from hypertension $(\mathrm{n}=166 ; 44.1 \% ; \mathrm{p}<0.05)$. Other comorbidities are listed in Table 2.

\section{Characteristics of OHCA}

Most of the CAs were witnessed ( $\mathrm{n}=268$; $77.7 \%$; $<$ 0.001). The initial rhythm in most of the patients was ventricular fibrillation or pulseless ventricular tachycardia (73.8\%). In more than half of the CA patients, CPR was initiated by paramedics (60.7\%; $\mathrm{p}<0.05)$. The mean time from CA to CPR was $7.2 \pm 8.6 \mathrm{~min}$ and from CA to ROSC $30.2 \pm 57.3$ min. Almost half of the CA survivors had a Glasgow Coma Scale (GCS) of 3 after the ROSC ( $n=147$; $49.5 \%$ ) and at the time of admission to hospital ( $\mathrm{n}=$ $=161 ; 47.4 \%)$. Twenty-one percent of survivors were in cardiogenic shock. The pre-cooling body temperature was $36.3 \pm 0.7^{\circ} \mathrm{C}$. In almost half of the patients, the time from CA to ROSC was greater than $20 \min (44.9 \%$; $p<0.05)$, and in $26.5 \%$ the CA to CPR time was greater than $10 \mathrm{~min}$. CA was not witnessed in $22.3 \%$ of patients. Non-shockable rhythm was initially registered in $20.8 \%$ of patients. One in $10(11.4 \%)$ patients experienced in-hospital CA.

Almost half of the patients scored 1 point in the MTH Scale $(\mathrm{p}<0.001)$. Only 1 patient scored 5 points $(0.4 \%)$. Details are shown in Table 3.

\section{Mortality}

In-hospital mortality was $18.6 \%$ (70 patients). Most deaths occurred after completion of the TTM procedure (53 out of 70 patients; $75.7 \%$ ), mainly due to multiorgan failure secondary to cardiac shock. The average time between admission and death was 15 days (range: $1-156$ days).
Table 2. Clinical characteristics on admission ( $n=376)$.

\begin{tabular}{|c|c|}
\hline Variable & Number (\%) \\
\hline Gender (male) & $301(80.1 \%)$ \\
\hline Hypertension & $166(44.1 \%)$ \\
\hline Past MI & $78(20.1 \%)$ \\
\hline Diabetes mellitus & $64(17.0 \%)$ \\
\hline Renal failure & $22(5.9 \%)$ \\
\hline History of stroke & $13(3.5 \%)$ \\
\hline Anemia & $8(2.3 \%)$ \\
\hline \multicolumn{2}{|l|}{ First rhythm of SCA: } \\
\hline VF/VT & $277(73.8 \%)$ \\
\hline Asystole & $74(19.6 \%)$ \\
\hline PEA & $25(6.5 \%)$ \\
\hline Time to CPR [min] & 7.2 \\
\hline Time to ROSC [min] & 30.2 \\
\hline \multicolumn{2}{|l|}{ Diagnosis on admission: } \\
\hline STEMI & $167(47.2)$ \\
\hline NSTEMI & $96(25.5)$ \\
\hline CAD & $12(3.3)$ \\
\hline DCM & $90(24.0)$ \\
\hline $\mathrm{HCM}$ & $11(3.0)$ \\
\hline \multicolumn{2}{|l|}{ GSC scale: } \\
\hline 3 points & $179(47.4)$ \\
\hline 4-6 points & $156(41.6)$ \\
\hline $7-8$ points & $41(11.0)$ \\
\hline Temperature on admission $\left[{ }^{\circ} \mathrm{C}\right]$ & 35.9 \\
\hline Cardiogenic shock & $79(21.0 \%)$ \\
\hline Primary $\mathrm{PCl}$ & $303(80.6 \%)$ \\
\hline Brain CT scan & $168(44.7 \%)$ \\
\hline Precooling with cold saline & $287(76.2 \%)$ \\
\hline \multicolumn{2}{|l|}{ Volume of cold saline for precooling $[\mathrm{mL}]$ : } \\
\hline$<1000$ & $13(4.6 \%)$ \\
\hline 1000 & $88(30.5 \%)$ \\
\hline 1500 & $90(31.4 \%)$ \\
\hline 2000 & $71(24.7 \%)$ \\
\hline$>2500$ & $25(8.8 \%)$ \\
\hline Time form ROSC do precooling [min] & 124.3 \\
\hline \multicolumn{2}{|l|}{ Cooling technique: } \\
\hline Intravascular & $88(23.4 \%)$ \\
\hline Surface cooling & $288(76.6 \%)$ \\
\hline
\end{tabular}

CAD — coronary artery disease; CPR — cardiopulmonary resuscitation; CT - computed tomography; DCM — dilated cardiomyopathy; GCS - Glasgow Coma Scale; HCM - hypertrophic cardiomyopathy; $\mathrm{MI}$ - myocardial infarction; NSTEMI - non-ST-segment elevation myocardial infarction; $\mathrm{PCl}$ - percutaneous coronary intervention; PEA - pulseless electrical activity; ROSC - return of spontaneous circulation; SCA — sudden cardiac arrest; STEMI ST-segment elevation myocardial infarction; VF — ventricular fibrillation; VT — ventricular tachycardia 
Table 3. Distribution of Mild Therapeutic Hypothermia (MTH) score in the registry.

\begin{tabular}{lcc}
\hline MTH Scale score & N & Percent \\
\hline 1 & 120 & $45.6 \%$ \\
2 & 103 & $39.2 \%$ \\
3 & 34 & $12.9 \%$ \\
4 & 4 & $1.9 \%$ \\
5 & 1 & $0.4 \%$ \\
\hline
\end{tabular}

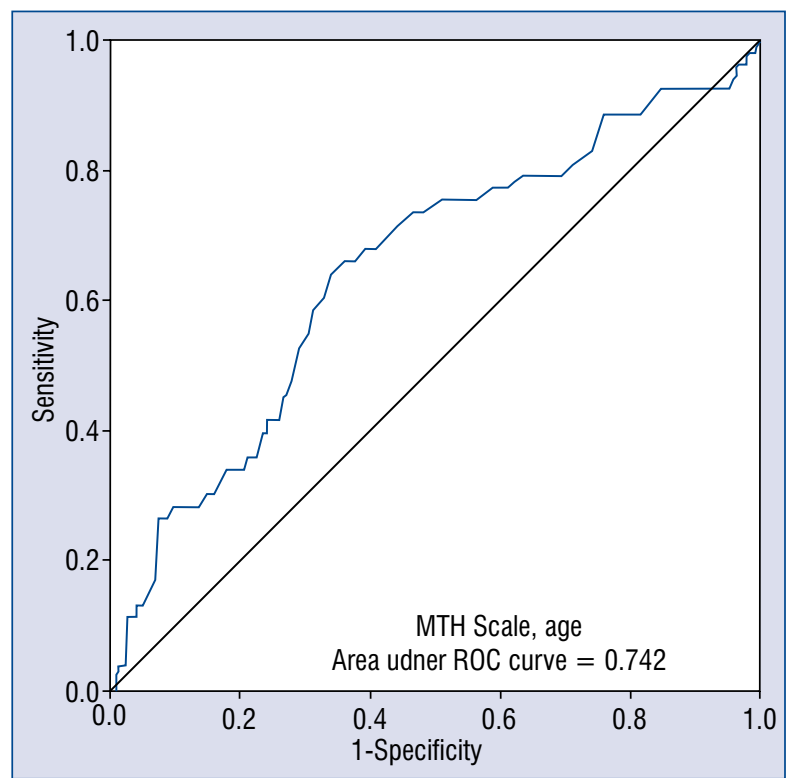

Figure 1. Predictive value of the Polish Hypothermia Registry Risk Score for prognosis of in-hospital mortality in post-cardiac arrest patients treated with targeted temperature management, expressed by the receiver operating characteristic (ROC) curve; MTH - Mild Therapeutic Hypothermia.

\section{Predictive value of the Polish Hypothermia Registry Risk Score}

Based on the results of the multivariate logistic regression model, a set of potential predictors of in-hospital mortality was identified. The greatest predictive values were confirmed for age and MTH scale score. To simplify the use of these predictors, a dedicated risk score was constructed and named the Polish Hypothermia Registry Risk Score (PHR-RS). The PHR-RS was calculated with the following equation: [(age [years] $\times 0.003)+($ score in MTH Scale $\times 0.11)-0.25] \times 100 \%$.

The predictive value of PHR-RS was expressed by an AUC of 0.74 , and it was the same after adding sex to the equation (Fig. 1).

\section{Discussion}

In this study, we described a simple model predicting a high probability of in-hospital death in patients after OHCA treated with TTM. The inputs of our model were the patient's age and MTH scale score, which includes time to CPR, time to ROSC, in-hospital localization of CA, whether the CA was witnessed or not, and whether the first rhythm was shockable. To the best of our knowledge, this is the first study to propose a scale enabling the prediction of the probability of in-hospital mortality in patients after OHCA with sufficient strength. Its use does not require any biochemical data or data from patient medical history and is based mainly on information from OHCA and CPR. There is a need for prognostic tools in this very demanding group of patients to help in decision-making about an escalation of medical treatment. PHR-RS may provide reliable data about patient in-hospital mortality risk, which would be useful when providing the information to patient family members.

Most studies on prediction factors in OHCA survivors date back to an era before the common use of TTM. In a meta-analysis of 79 studies involving 142,740 patients after OHCA, the pooled survival rate to hospital discharge was $7.6 \%$ and failed to improve significantly despite technological advances. This meta-analysis included studies carried out between 1950 and 2008, when TTM was not commonly used. The influence of TTM was not evaluated, but the authors reported that patients with witnessed OHCA, ventricular fibrillation/ventricular tachycardia (VF/VT), and in whom ROSC was achieved, had a greater chance of being discharged from the hospital [4]. Early $\mathrm{CPR} /$ defibrillation performed by a by-stander or members of a medical team, CA occurrence outside the home, the presence of initial shockable rhythms, and quick ROSC were shown to be better prognostic factors in several other studies [5-8]. Wibrandt et al. [6] reported that VT/VF as initial rhythm, the cardiac etiology of OHCA and time to ROSC $<20$ min were strong predictors of both survival and favourable neurological outcomes. In a recent Polish study on pre-hospital death predictors in OHCA victims, Gach et al. [9] reported that univariate analysis indicated 6 predictors of death: OHCA without witnesses, OHCA in a public place, no bystander CPR, no bystander cardiac massage, initial diagnosis of non-shockable cardiac rhythm and the amount of drugs used for CPR. However, logistic regression confirmed that only the lack of bystander cardiac massage and non-shockable 
rhythm were independent determinants of prehospital death [9]. Among other recent reports, Aldhoon et al. [10] examined the factors affecting 1 -month outcomes in 46 OHCA survivors treated with hypothermia. In this study, patients eventually discharged in good neurological condition were younger and had lower lactate level on admission. In addition, the underlying cause of $\mathrm{CA}$ was acute myocardial infarction [10].

The data on the influence of sex on survival are conflicting, with some studies reporting better survival in women $[5,11]$, another in men [12], and in some no difference between the sexes was observed $[13,14]$. The literature is lacking in studies evaluating how comorbidities influence survival. Only Fabbri et al. [15] reported that no history of hypertension, diabetes, myocardial infarction, or congestive heart failure were positive outcome predictors after OHCA. It is known that lactate level [10] and $\mathrm{pH}$ [8] are important in terms of survival prognosis after OHCA. Whittaker et al. [8] used a multivariate logistic regression analysis to demonstrate that an initial $\mathrm{pH} \leq 7.1$, among other factors, was a predictor of in-hospital mortality after OHCA. Aldhoon et al. [10] showed that OHCA survivors who underwent TTM with a favourable outcome had lower lactate levels on admission and after $24 \mathrm{~h}$ of hospital treatment. The level of acidosis is often interpreted as a measurement of the length and adequacy of CPR, increasingly more studies emphasize the importance of this parameter [16]. The present model did not include biochemical parameters because the aim was to create a quick and easy to use scale that could be calculated in the emergency room even prior to obtaining blood test results. The main limitation of the present study is a lack of external validation of the proposed risk score. An international registry would allow for the confirmation of the predictive value in a multinational patient population treated according to various local protocols. Also, bias related to the relative weight of each center and the unbalanced distribution of the TTM procedure could not excluded. However, all centers used one standardised TTM protocol. Furthermore, there was a relatively small group of patients included in the study. Finally, the MTH score was assigned based on expert opinions and has never been validated by any clinical data set.

Krawczyk et al. [17] emphasised the need for discussion and education when they reported that the TTM procedure in Polish ICUs is underused. The most common limitations include a lack of dedicated devices, logistical issues and a misunderstanding or misinterpretation of published evidence. Herein, is hypothesized that the PHR-RS, as a simple predictive model, may help in TTM implementation.

Another issue is the neurological prognostication in post-CA survivors. The SPAME trial revealed that most large university hospitals in Europe had a standard operating protocol for TTM. However, only a minority used a standard operating protocol for neurological prognostication, giving electroencephalography and brain CT scans the highest priority [18]. PHR-RS was not evaluated as a neurological prognostication tool.

\section{Conclusions}

The PHR-RS is a simple tool that may be useful in the prediction of the in-hospital mortality in OHCA patients treated with TTM. The PHR-RS was created based on the multicenter register and includes age and MTH score.

\section{Acknowledgements}

This analysis would not have been possible without the great enthusiasm and support of a number of supporters and academic friends. We would like to thank the following physicians who helped in the development of the Polish Hypothermia Registry and researchers who actively reported cases to the database: Rafał Depukat, Aleksander Zeliaś, Tomasz Adamek, Bożena Sobkowicz, Hanna Rdzanek, Andrzej Gosk, Andrzej Tarchalski, Bartosz Kudliński, Jerzy Węgielnik, Jakub Kołowski, Janusz Kudlicki, Wojciech Golecki, Konrad Koza, Krzysztof Sys, Leszek Pawłowski, Łukasz Lewicki, Monika Lica, Maciej Gawor, Magdalena Czajkowska, Marek Banaszewski, Katarzyna Żukowska, Katarzyna Szepietowska, Marta Dobrowolska-Jaśkiewicz, Michał Zabojszcz, Miłosz Jankowski, Michał Walczewski, Michał Nedoszytko, Michał Dworzyński, Paweł Andruszkiewicz, Piotr Nowakowski, Paweł Spychalski, Piotr Woźniak, Robert Ryczek, Wojciech Burkot, Wiktor Sułkowski, Tomasz Wiśniewski, Zbigniew Brachaczek, Zbigniew Żyła, Andrzej Świątkowski, and Dariusz Dudek.

Conflict of interest: Beata Średniawa and Janina Stępińska participated in studies conducted by ZOLL Corporation. Łukasz Koltowski and Grzegorz Opolski received a speaker fee by BARD POLSKA. The rest of the authors declare no conflict of interest. 


\section{References}

1. Lick CJ, Aufderheide TP, Niskanen RA, et al. Take Heart America: a comprehensive, community-wide, systems-based approach to the treatment of cardiac arrest. Crit Care Med. 2011; 39(1): 26-33, doi: 10.1097/CCM.0b013e3181fa7ce4, indexed in Pubmed: 20890185.

2. Ishikawa S, Niwano S, Imaki R, et al. Usefulness of a simple prognostication score in prediction of the prognoses of patients with out-of-hospital cardiac arrests. Int Heart J. 2013; 54(6): 362-370, indexed in Pubmed: 24309445.

3. Skrifvars MB, Varghese B, Parr MJ. Survival and outcome prediction using the Apache III and the out-of-hospital cardiac arrest $(\mathrm{OHCA})$ score in patients treated in the intensive care unit (ICU) following out-of-hospital, in-hospital or ICU cardiac arrest. Resuscitation. 2012; 83(6): 728-733, doi: 10.1016/j.resuscitation.2011.11.036, indexed in Pubmed: 22281225.

4. Sasson C, Rogers MAM, Dahl J, et al. Predictors of survival from out-of-hospital cardiac arrest: a systematic review and metaanalysis. Circ Cardiovasc Qual Outcomes. 2010; 3(1): 63-81, doi: 10.1161/CIRCOUTCOMES.109.889576, indexed in Pubmed: 20123673.

5. Adielsson A, Hollenberg J, Karlsson T, et al. Increase in survival and bystander CPR in out-of-hospital shockable arrhythmia: bystander CPR and female gender are predictors of improved outcome. Experiences from Sweden in an 18-year perspective. Heart. 2011; 97(17): 1391-1396, doi: 10.1136/hrt.2011.222711, indexed in Pubmed: 21715444.

6. Wibrandt I, Norsted K, Schmidt H, et al. Predictors for outcome among cardiac arrest patients: the importance of initial cardiac arrest rhythm versus time to return of spontaneous circulation, a retrospective cohort study. BMC Emerg Med. 2015; 15: 3, doi: 10.1186/s12873-015-0028-3, indexed in Pubmed: 25648841.

7. Iqbal MB, Al-Hussaini A, Rosser G, et al. Predictors of survival and favorable functional outcomes after an out-of-hospital cardiac arrest in patients systematically brought to a dedicated heart attack center (from the Harefield Cardiac Arrest Study). Am J Cardiol. 2015; 115(6): 730-737, doi: 10.1016/j.amjcard.2014.12.033, indexed in Pubmed: 25644852.

8. Whittaker A, Lehal M, Calver AL, et al. Predictors of inhospital mortality following out-of-hospital cardiac arrest: insights from a single-centre consecutive case series. Postgrad Med J. 2016; 92(1087): 250-254, doi: 10.1136/postgradmedj-2015-133575, indexed in Pubmed: 26739845.
9. Gach D, Nowak JU, Krzych ŁJ. Determinants of unfavorable prognosis for out-of-hospital sudden cardiac arrest in Bielsko-Biala district. Kardiochir Torakochirurgia Pol. 2016; 13(3): 217-223, doi: 10.5114/kitp.2016.62195, indexed in Pubmed: 27785135.

10. Aldhoon B, Melenovsky V, Kettner J, et al. Clinical predictors of outcome in survivors of out-of-hospital cardiac arrest treated with hypothermia. Cor et Vasa. 2012; 54(2): e68-e75, doi: 10.1016/j.crvasa.2012.01.005.

11. Bougouin W, Mustafic H, Marijon E, et al. Gender and survival after sudden cardiac arrest: a systematic review and meta-analysis. Resuscitation. 2015; 94: 55-60, doi: 10.1016/j.resuscitation.2015.06.018, indexed in Pubmed: 26143159.

12. Bosson N, Kaji AH, Fang A, et al. Sex differences in survival from out-of-hospital cardiac arrest in the era of regionalized systems and advanced post-resuscitation care. J Am Heart Assoc. 2016; 5(9), doi: 10.1161/JAHA.116.004131, indexed in Pubmed: 27633392.

13. Pachys G, Kaufman N, Bdolah-Abram T, et al. Predictors of longterm survival after out-of-hospital cardiac arrest: the impact of Activities of Daily Living and Cerebral Performance Category scores. Resuscitation. 2014; 85(8): 1052-1058, doi: 10.1016/j. resuscitation.2014.03.312, indexed in Pubmed: 24727137.

14. Winther-Jensen M, Kjaergaard J, Wanscher M, et al. No difference in mortality between men and women after out-of-hospital cardiac arrest. Resuscitation. 2015; 96: 78-84, doi: 10.1016/j. resuscitation.2015.06.030.

15. Fabbri A, Marchesini G, Spada M, et al. Monitoring intervention programmes for out-of-hospital cardiac arrest in a mixed urban and rural setting. Resuscitation. 2006; 71(2): 180-187, doi: 10.1016/j.resuscitation.2006.04.003, indexed in Pubmed: 16982124.

16. Adnet F, Triba MN, Borron SW, et al. Cardiopulmonary resuscitation duration and survival in out-of-hospital cardiac arrest patients. Resuscitation. 2017; 111: 74-81, doi: 10.1016/j.resuscitation.2016.11.024, indexed in Pubmed: 27987396.

17. Krawczyk P, Tarczyńska A, Dziadek G, et al. Implementation of targeted temperature management after cardiac arrest in Polish intensive care units. What has changed in the last five years? Kardiol Pol. 2017; 75(7): 689-697, doi: 10.5603/KP.a2017.0073, indexed in Pubmed: 28553848.

18. Storm C, Nee J, Sunde K, et al. A survey on general and temperature management of post cardiac arrest patients in large teaching and university hospitals in 14 European countries. The SPAME trial results. Resuscitation. 2017; 116: 84-90, doi: 10.1016/j.resuscitation.2017.03.038, indexed in Pubmed: 28377294. 